\title{
The 12-Membered Ring Carbides with Stabilization of Actinide Atoms
}

\author{
Ke Zhou, ${ }^{1}$ Yang-Yang Zhang, ${ }^{2}$ Xiao-Tong Chen, ${ }^{3}$ Shu-Xian $\mathrm{Hu}^{* 4,5}$
}

${ }^{1}$ College of Chemistry and Environmental Science \& Shaanxi Key Laboratory of Catalysis \& Institute of Theoretical and Computational Chemistry, Shaanxi University of Technology, 723000, Hanzhong, China.

${ }^{2}$ Department of Chemistry \& Key Laboratory of Organic Optoelectronics, Tsinghua University, Beijing 100084, China.

${ }^{3}$ Institute of Nuclear and New Energy Technology \& Collaborative Innovation Center of Advanced Nuclear Energy Technology, Tsinghua University, Beijing100084, China.

${ }^{4}$ Department of Physics, University of Science and Technology Beijing, Beijing 100083, China.

${ }^{5}$ Beijing Computational Science Research Center, Beijing 100193, China.

*Corresponding Authors: hushuxian@csrc.ac.cn (S.-X.H.)

\section{Table of Contents}

Table S1. The relative energies $(\Delta \mathrm{E} 1, \mathrm{kcal} / \mathrm{mol})$ compared with the most stable isomers and the relative energies $(\Delta \mathrm{E} 2, \mathrm{kcal} / \mathrm{mol})$ between different multiplicities of each isomer for $\left[\mathrm{C}_{12}\right](\mathrm{A})$ at B3PW91/6-31+G(d) and PBE0/6-31+G(d) two levels.

Pages S2-S4

Table S2. The relative energies $(\Delta \mathrm{E} 1, \mathrm{kcal} / \mathrm{mol})$ compared with the most stable isomers and the relative energies $(\Delta \mathrm{E} 2, \mathrm{kcal} / \mathrm{mol})$ between different multiplicities of each isomer for $\mathrm{ThC}_{12}$ (B) at B3PW91 and PBE0/(Th: SDD; C: 6-31+G(d)) two levels.

Pages S5-S7

Table S3. The relative energies ( $\Delta \mathrm{E} 1, \mathrm{kcal} / \mathrm{mol})$ compared to the most stable isomers and the relative energies $(\triangle \mathrm{E} 2, \mathrm{kcal} / \mathrm{mol})$ between different multiplicities of each isomer for $\mathrm{UC}_{12}(\mathbf{C})$ at B3PW91 and PBE0/(U: SDD; C: 6-31+G(d)) two levels.

Table S4. Relative energies ( $\Delta \mathrm{E}, \mathrm{kcal} / \mathrm{mol})$ for different spin states of $\mathbf{1 B}$ and $\mathbf{1 C}$.

Pages S12

Table S5. EDA analyses for $\mathrm{An} / \mathrm{An}^{4+}+\left[\mathrm{C}_{12}\right] /\left[\mathrm{C}_{12}\right]^{4-} \rightarrow\left[\mathrm{AnC}_{12}\right](\mathrm{An}=\mathrm{Th}, \mathrm{U})$ at the PBE0/TZ2P level of theory.

Pages S13

Table S6. The Mayer, Gopinathan-Jug and Nalewajski-Mrozek bond orders for $\mathrm{C}-\mathrm{C}$ and $\mathrm{An}-\mathrm{C}$ bonds, and charges on the $\mathrm{An}$ and $\mathrm{C}$ of the 1A, 1B and $1 \mathrm{C}$ at the PBE0/TZ2P level.

Page S14

Figure S1. The structures, partial labels, full point group, and relative energies in $\mathrm{kcal} / \mathrm{mol}$ for the selected isomers of $\left[\mathrm{C}_{12}\right](\mathrm{A})$ at PBE0/6-31+G(d) level.

Page S15

Figure S2. The structures of most stable spin multiplicities and relative energies in $\mathrm{kcal} / \mathrm{mol}$ for the selected isomers of $\mathrm{ThC}_{12}$ (B) at PBE0/(Th: SDD; C: 6-31+G(d)) level.

Page S16

Figure S3. The structures of most stable spin multiplicities, and relative energies in $\mathrm{kcal} / \mathrm{mol}$ for the selected isomers of $\mathrm{UC}_{12}(\mathbf{C})$ at PBE0/(U: SDD; C: $\left.6-31+\mathrm{G}(\mathrm{d})\right)$ level. 
Table S1. The relative energies $(\Delta \mathrm{E} 1, \mathrm{kcal} / \mathrm{mol})$ compared with the most stable isomers and the relative energies $(\Delta \mathrm{E} 2, \mathrm{kcal} / \mathrm{mol})$ between different multiplicities of each isomer for $\left[\mathrm{C}_{12}\right](\mathbf{A})$ at B3PW91/6-31+G(d) and PBE0/6$31+\mathrm{G}(\mathrm{d})$ two levels.

\begin{tabular}{|c|c|c|c|c|c|c|}
\hline \multirow{2}{*}[\mathrm{C}_{12}]{} & \multirow{2}{*}{$2 \mathrm{~S}+1$} & \multirow{2}{*}{ State } & \multicolumn{2}{|c|}{ B3PW91 } & \multicolumn{2}{|c|}{ PBE0 } \\
\hline & & & $\Delta \mathrm{E} 1$ & $\Delta \mathrm{E} 2$ & $\Delta \mathrm{E} 1$ & $\Delta \mathrm{E} 2$ \\
\hline $1 \mathrm{~A}$ & 1 & ${ }^{1} \mathrm{~A}_{1}{ }^{\prime}$ & 0.00 & 0.00 & 0.00 & 0.00 \\
\hline $1 \mathrm{~A}$ & 3 & ${ }^{3} \mathrm{~A}^{\prime}$ & 5.02 & 5.02 & 5.47 & 5.47 \\
\hline $2 \mathrm{~A}$ & 1 & ${ }^{1} \Sigma$ & 32.26 & 6.49 & 37.41 & 9.85 \\
\hline $2 \mathrm{~A}$ & 3 & ${ }^{3} \Sigma$ & 25.76 & 0.00 & 27.56 & 0.00 \\
\hline $2 \mathrm{~A}$ & 5 & ${ }^{5} \mathrm{~A}$ & 74.73 & 48.97 & 78.80 & 51.24 \\
\hline $3 \mathrm{~A}$ & 1 & ${ }^{1} \mathrm{~A}$ & 61.09 & 0.00 & 57.80 & 0.00 \\
\hline $3 \mathrm{~A}$ & 3 & ${ }^{3} \mathrm{~A}$ & 83.84 & 22.75 & 80.93 & 23.14 \\
\hline $4 \mathrm{~A}$ & 1 & ${ }^{1} \mathrm{~A}$ & 63.34 & 0.00 & 60.19 & 0.00 \\
\hline $4 \mathrm{~A}$ & 3 & ${ }^{3} \mathrm{~A}$ & 74.41 & 11.07 & 70.64 & 10.46 \\
\hline $5 \mathrm{~A}$ & 1 & ${ }^{1} \mathrm{~A}$ & 64.06 & 0.00 & 60.97 & 0.00 \\
\hline $5 \mathrm{~A}$ & 3 & ${ }^{3} \mathrm{~A}$ & 81.55 & 17.49 & 78.28 & 17.31 \\
\hline $6 \mathrm{~A}$ & 1 & ${ }^{1} \mathrm{~A}$ & 71.91 & 0.00 & 67.59 & 0.00 \\
\hline $6 \mathrm{~A}$ & 3 & ${ }^{3} \mathrm{~A}$ & 97.55 & 25.64 & 93.63 & 26.04 \\
\hline $7 \mathrm{~A}$ & 1 & ${ }^{1} \mathrm{~A}$ & 79.98 & 0.00 & 72.55 & 0.00 \\
\hline $7 \mathrm{~A}$ & 3 & ${ }^{3} \mathrm{~A}$ & 86.69 & 6.71 & 81.02 & 8.47 \\
\hline $8 \mathrm{~A}$ & 1 & ${ }^{1} \mathrm{~A}$ & 84.49 & 0.00 & 80.55 & 0.00 \\
\hline $8 \mathrm{~A}$ & 3 & ${ }^{3} \mathrm{~A}$ & 92.57 & 8.08 & 86.88 & 6.33 \\
\hline $9 \mathrm{~A}$ & 1 & ${ }^{1} \mathrm{~A}$ & 87.09 & 0.00 & 84.54 & 0.00 \\
\hline $9 \mathrm{~A}$ & 3 & ${ }^{3} \mathrm{~A}$ & 96.95 & 9.86 & 86.60 & 2.06 \\
\hline $10 \mathrm{~A}$ & 1 & ${ }^{1} \mathrm{~A}_{1}$ & 90.82 & 0.00 & 85.31 & 0.00 \\
\hline $10 \mathrm{~A}$ & 3 & ${ }^{3} \mathrm{~A}$ & 111.95 & 21.13 & 105.41 & 20.10 \\
\hline $11 \mathrm{~A}$ & 1 & ${ }^{1} \mathrm{~A}$ & 103.70 & 0.00 & 99.03 & 0.00 \\
\hline $11 \mathrm{~A}$ & 3 & ${ }^{3} \mathrm{~A}$ & 119.52 & 15.82 & 114.11 & 15.07 \\
\hline $12 \mathrm{~A}$ & 1 & ${ }^{1} \mathrm{~A}$ & 110.96 & 0.00 & 106.51 & 0.00 \\
\hline $12 \mathrm{~A}$ & 3 & ${ }^{3} \mathrm{~A}$ & 121.46 & 10.51 & 116.25 & 9.74 \\
\hline $13 \mathrm{~A}$ & 1 & ${ }^{1} \mathrm{~A}$ & 117.63 & 0.00 & 106.86 & 0.00 \\
\hline $13 \mathrm{~A}$ & 3 & ${ }^{3} \mathrm{~A}$ & 128.02 & 10.39 & 113.78 & 6.91 \\
\hline
\end{tabular}




\begin{tabular}{|c|c|c|c|c|c|c|}
\hline $14 \mathrm{~A}$ & 1 & ${ }^{1} \mathrm{~A}$ & 116.73 & 0.00 & 107.22 & 0.00 \\
\hline $14 \mathrm{~A}$ & 3 & ${ }^{3} \mathrm{~A}$ & 124.85 & 8.12 & 113.69 & 6.47 \\
\hline $15 \mathrm{~A}$ & 1 & ${ }^{1} \mathrm{~A}$ & 116.38 & 0.00 & 108.77 & 0.00 \\
\hline $15 \mathrm{~A}$ & 3 & ${ }^{3} \mathrm{~A}$ & 131.16 & 14.78 & 122.73 & 13.96 \\
\hline $16 \mathrm{~A}$ & 1 & ${ }^{1} \mathrm{~A}$ & 117.98 & 0.00 & 112.84 & 0.00 \\
\hline $16 \mathrm{~A}$ & 3 & ${ }^{3} \mathrm{~A}$ & 120.71 & 2.73 & 114.16 & 1.32 \\
\hline $17 \mathrm{~A}$ & 1 & ${ }^{1} \mathrm{~A}$ & 123.10 & 0.00 & 112.85 & 0.00 \\
\hline $17 \mathrm{~A}$ & 3 & ${ }^{3} \mathrm{~A}$ & 134.01 & 10.91 & 121.36 & 8.50 \\
\hline $18 \mathrm{~A}$ & 1 & ${ }^{1} \mathrm{~A}$ & 156.53 & 30.79 & 146.93 & 33.16 \\
\hline $18 \mathrm{~A}$ & 3 & ${ }^{3} \mathrm{~A}$ & 125.74 & 0.00 & 113.78 & 0.00 \\
\hline $18 \mathrm{~A}$ & 5 & ${ }^{5} \mathrm{~A}$ & 147.22 & 21.48 & 134.58 & 20.81 \\
\hline $19 \mathrm{~A}$ & 1 & ${ }^{1} \mathrm{~A}$ & 114.20 & 0.00 & 116.90 & 0.00 \\
\hline $19 \mathrm{~A}$ & 3 & ${ }^{3} \mathrm{~A}$ & 125.76 & 11.57 & 129.27 & 12.37 \\
\hline $20 \mathrm{~A}$ & 1 & ${ }^{1} \mathrm{~A}^{\prime}$ & 123.40 & 0.00 & 116.90 & 0.00 \\
\hline $20 \mathrm{~A}$ & 3 & ${ }^{3} \mathrm{~A}$ & 137.90 & 14.49 & 129.66 & 12.76 \\
\hline $21 \mathrm{~A}$ & 1 & ${ }^{1} \mathrm{~A}$ & 130.02 & 1.85 & 124.53 & 2.91 \\
\hline $21 \mathrm{~A}$ & 3 & ${ }^{3} \mathrm{~A}$ & 128.17 & 0.00 & 121.62 & 0.00 \\
\hline $21 \mathrm{~A}$ & 5 & ${ }^{5} \mathrm{~A}$ & 137.89 & 9.72 & 130.01 & 8.39 \\
\hline $22 \mathrm{~A}$ & 1 & ${ }^{1} \mathrm{~A}$ & 128.50 & 0.00 & 121.93 & 0.00 \\
\hline $22 \mathrm{~A}$ & 3 & ${ }^{3} \mathrm{~A}$ & 136.04 & 7.55 & 128.45 & 6.51 \\
\hline $23 \mathrm{~A}$ & 1 & ${ }^{1} \mathrm{~A}$ & 140.42 & 0.00 & 128.46 & 0.00 \\
\hline $23 \mathrm{~A}$ & 3 & ${ }^{3} \mathrm{~A}$ & 155.22 & 14.80 & 143.87 & 15.41 \\
\hline $24 \mathrm{~A}$ & 1 & ${ }^{1} \mathrm{~A}$ & 140.83 & 0.00 & 133.60 & 0.00 \\
\hline $24 \mathrm{~A}$ & 3 & ${ }^{3} \mathrm{~A}$ & 146.94 & 6.11 & 137.87 & 4.27 \\
\hline $25 \mathrm{~A}$ & 1 & ${ }^{1} \mathrm{~A}$ & 146.85 & 2.78 & 140.22 & 4.85 \\
\hline $25 \mathrm{~A}$ & 3 & ${ }^{3} \mathrm{~A}$ & 144.06 & 0.00 & 135.37 & 0.00 \\
\hline $25 \mathrm{~A}$ & 5 & ${ }^{5} \mathrm{~A}$ & 151.82 & 7.76 & 143.45 & 8.08 \\
\hline $26 \mathrm{~A}$ & 1 & ${ }^{1} \mathrm{~A}^{\prime}$ & 145.41 & 0.00 & 139.45 & 0.26 \\
\hline $26 \mathrm{~A}$ & 3 & ${ }^{3} \mathrm{~A}$ & 146.91 & 1.50 & 139.19 & 0.00 \\
\hline $27 \mathrm{~A}$ & 1 & ${ }^{1} \mathrm{~A}^{\prime}$ & 143.34 & 0.00 & 139.39 & 0.00 \\
\hline $27 \mathrm{~A}$ & 3 & ${ }^{3} \mathrm{~A}$ & 150.35 & 7.02 & 145.15 & 5.76 \\
\hline $28 \mathrm{~A}$ & 1 & ${ }^{1} \mathrm{~A}$ & 148.31 & 0.00 & 142.51 & 0.00 \\
\hline
\end{tabular}




\begin{tabular}{|c|c|c|c|c|c|c|}
\hline $28 \mathrm{~A}$ & 3 & ${ }^{3} \mathrm{~A}$ & 164.89 & 16.58 & 157.83 & 15.33 \\
\hline $29 \mathrm{~A}$ & 1 & ${ }^{1} \mathrm{~A}^{\prime}$ & 155.70 & 0.00 & 146.76 & 0.00 \\
\hline $29 \mathrm{~A}$ & 3 & ${ }^{3} \mathrm{~A}$ & 161.80 & 6.10 & 152.40 & 5.63 \\
\hline $30 \mathrm{~A}$ & 1 & ${ }^{1} \mathrm{~A}$ & 158.48 & 2.94 & 159.79 & 1.57 \\
\hline $30 \mathrm{~A}$ & 3 & ${ }^{3} \mathrm{~A}$ & 155.54 & 0.00 & 158.22 & 0.00 \\
\hline $30 \mathrm{~A}$ & 5 & ${ }^{5} \mathrm{~A}$ & 181.47 & 25.93 & 179.09 & 20.87 \\
\hline
\end{tabular}


Table S2. The relative energies $(\Delta \mathrm{E} 1, \mathrm{kcal} / \mathrm{mol})$ compared with the most stable isomers and the relative energies $(\Delta \mathrm{E} 2, \mathrm{kcal} / \mathrm{mol})$ between different multiplicities of each isomer for $\mathrm{ThC}_{12}(\mathrm{~B})$ at B3PW91/(Th: SDD; C: 6-31+G(d)) and PBE0/(Th: SDD; C: 6-31+G(d)) two levels.

\begin{tabular}{|c|c|c|c|c|c|c|}
\hline \multirow{2}{*}{$\mathrm{ThC}_{12}$} & \multirow{2}{*}{$2 \mathrm{~S}+1$} & \multirow{2}{*}{ State } & \multicolumn{2}{|c|}{ B3PW91 } & \multicolumn{2}{|c|}{ PBE0 } \\
\hline & & & $\Delta \mathrm{E} 1$ & $\Delta \mathrm{E} 2$ & $\Delta \mathrm{E} 1$ & $\Delta \mathrm{E} 2$ \\
\hline $1 \mathrm{~B}$ & 1 & ${ }^{1} \mathrm{~A}$ & 0.00 & 0.00 & 0.00 & 0.00 \\
\hline $1 \mathrm{~B}$ & 3 & ${ }^{3} \mathrm{~A}$ & 22.17 & 22.17 & 22.34 & 22.34 \\
\hline $2 \mathrm{~B}$ & 1 & ${ }^{1} \mathrm{~A}$ & 48.72 & 0.00 & 52.12 & 0.00 \\
\hline $2 \mathrm{~B}$ & 3 & ${ }^{3} \mathrm{~A}$ & 72.97 & 24.26 & 80.12 & 28.00 \\
\hline $2 \mathrm{~B}$ & 5 & ${ }^{5} \mathrm{~A}$ & 93.18 & 44.46 & 99.88 & 47.76 \\
\hline $3 B$ & 1 & ${ }^{1} \mathrm{~A}$ & 59.55 & 0.00 & 63.02 & 0.00 \\
\hline $3 B$ & 3 & ${ }^{3} \mathrm{~A}$ & 101.36 & 41.81 & 104.65 & 41.64 \\
\hline $4 B$ & 1 & ${ }^{1} \mathrm{~A}$ & 71.99 & 1.66 & 75.16 & 3.71 \\
\hline $4 B$ & 3 & ${ }^{3} \mathrm{~A}$ & 70.33 & 0.00 & 71.45 & 0.00 \\
\hline $4 B$ & 5 & ${ }^{5} \mathrm{~A}$ & 103.05 & 32.72 & 103.89 & 32.44 \\
\hline $5 \mathrm{~B}$ & 1 & ${ }^{1} \mathrm{~A}$ & 71.38 & 0.00 & 75.31 & 0.00 \\
\hline $5 B$ & 3 & ${ }^{3} \mathrm{~A}$ & 89.11 & 17.73 & 93.57 & 18.26 \\
\hline $6 \mathrm{~B}$ & 1 & ${ }^{1} \mathrm{~A}$ & 82.94 & 0.00 & 81.46 & 1.02 \\
\hline $6 \mathrm{~B}$ & 3 & ${ }^{3} \mathrm{~A}$ & 83.63 & 0.68 & 80.44 & 0.00 \\
\hline $6 \mathrm{~B}$ & 5 & ${ }^{5} \mathrm{~A}$ & 115.00 & 32.05 & 111.82 & 31.38 \\
\hline $7 \mathrm{~B}$ & 1 & ${ }^{1} \mathrm{~A}$ & 93.09 & 0.00 & 89.43 & 0.00 \\
\hline $7 \mathrm{~B}$ & 3 & ${ }^{3} \mathrm{~A}$ & 116.22 & 23.14 & 112.23 & 22.80 \\
\hline $8 B$ & 1 & ${ }^{1} \mathrm{~A}$ & 100.72 & 0.00 & 97.73 & 0.00 \\
\hline $8 B$ & 3 & ${ }^{3} \mathrm{~A}$ & 113.47 & 12.75 & 110.19 & 12.46 \\
\hline 9B & 1 & ${ }^{1} \mathrm{~A}$ & 106.80 & 5.38 & 101.90 & 1.64 \\
\hline 9B & 3 & ${ }^{3} \mathrm{~A}$ & 101.43 & 0.00 & 100.26 & 0.00 \\
\hline $9 B$ & 5 & ${ }^{5} \mathrm{~A}$ & 102.70 & 1.27 & 105.00 & 4.74 \\
\hline $10 \mathrm{~B}$ & 1 & ${ }^{1} \mathrm{~A}$ & 116.09 & 10.08 & 114.11 & 11.13 \\
\hline 10B & 3 & ${ }^{3} \mathrm{~A}$ & 106.02 & 0.00 & 102.97 & 0.00 \\
\hline $10 \mathrm{~B}$ & 5 & ${ }^{5} \mathrm{~A}$ & 119.25 & 13.23 & 116.34 & 13.36 \\
\hline $11 \mathrm{~B}$ & 1 & ${ }^{1} \mathrm{~A}$ & 103.80 & 1.24 & 111.46 & 6.61 \\
\hline
\end{tabular}




\begin{tabular}{|c|c|c|c|c|c|c|}
\hline $11 \mathrm{~B}$ & 3 & ${ }^{3} \mathrm{~A}$ & 102.57 & 0.00 & 104.85 & 0.00 \\
\hline $11 \mathrm{~B}$ & 5 & ${ }^{5} \mathrm{~A}$ & 109.12 & 6.55 & 105.21 & 0.36 \\
\hline $12 \mathrm{~B}$ & 1 & ${ }^{1} \mathrm{~A}$ & 110.75 & 0.00 & 106.91 & 0.00 \\
\hline $12 \mathrm{~B}$ & 3 & ${ }^{3} \mathrm{~A}$ & 118.98 & 8.23 & 114.52 & 7.61 \\
\hline $13 \mathrm{~B}$ & 1 & ${ }^{1} \mathrm{~A}$ & 124.24 & 0.00 & 119.28 & 0.00 \\
\hline $13 \mathrm{~B}$ & 3 & ${ }^{3} \mathrm{~A}$ & 126.95 & 2.71 & 120.73 & 1.45 \\
\hline $14 \mathrm{~B}$ & 1 & ${ }^{1} \mathrm{~A}$ & 127.44 & 0.00 & 120.94 & 0.00 \\
\hline $14 \mathrm{~B}$ & 3 & ${ }^{3} \mathrm{~A}$ & 131.80 & 4.37 & 125.85 & 4.91 \\
\hline $15 \mathrm{~B}$ & 1 & ${ }^{1} \mathrm{~A}$ & 124.50 & 0.00 & 122.66 & 0.00 \\
\hline $15 \mathrm{~B}$ & 3 & ${ }^{3} \mathrm{~A}$ & 139.48 & 14.98 & 137.25 & 14.59 \\
\hline $16 \mathrm{~B}$ & 1 & ${ }^{1} \mathrm{~A}$ & 128.43 & 0.00 & 124.33 & 0.00 \\
\hline $16 \mathrm{~B}$ & 3 & ${ }^{3} \mathrm{~A}$ & 131.89 & 3.46 & 127.35 & 3.03 \\
\hline $17 \mathrm{~B}$ & 1 & ${ }^{1} \mathrm{~A}$ & 133.10 & 0.00 & 125.11 & 0.00 \\
\hline $17 \mathrm{~B}$ & 3 & ${ }^{3} \mathrm{~A}$ & 148.14 & 15.05 & 139.69 & 14.57 \\
\hline $18 \mathrm{~B}$ & 1 & ${ }^{1} \mathrm{~A}$ & 137.69 & 10.23 & 136.85 & 11.17 \\
\hline $18 \mathrm{~B}$ & 3 & ${ }^{3} \mathrm{~A}$ & 127.46 & 0.00 & 125.67 & 0.00 \\
\hline $18 \mathrm{~B}$ & 5 & ${ }^{5} \mathrm{~A}$ & 145.75 & 18.29 & 143.30 & 17.63 \\
\hline $19 \mathrm{~B}$ & 1 & ${ }^{1} \mathrm{~A}$ & 138.94 & 0.00 & 132.61 & 0.00 \\
\hline 19B & 3 & ${ }^{3} \mathrm{~A}$ & 149.61 & 10.67 & 143.34 & 10.73 \\
\hline $20 \mathrm{~B}$ & 1 & ${ }^{1} \mathrm{~A}$ & 139.85 & 0.00 & 135.07 & 0.00 \\
\hline $20 \mathrm{~B}$ & 3 & ${ }^{3} \mathrm{~A}$ & 154.70 & 14.85 & 150.13 & 15.06 \\
\hline $21 \mathrm{~B}$ & 1 & ${ }^{1} \mathrm{~A}$ & 141.71 & 3.17 & 138.06 & 2.99 \\
\hline $21 \mathrm{~B}$ & 3 & ${ }^{3} \mathrm{~A}$ & 138.54 & 0.00 & 135.08 & 0.00 \\
\hline $21 \mathrm{~B}$ & 5 & ${ }^{5} \mathrm{~A}$ & 166.13 & 27.59 & 162.42 & 27.34 \\
\hline $22 \mathrm{~B}$ & 1 & ${ }^{1} \mathrm{~A}$ & 137.22 & 0.00 & 135.41 & 0.00 \\
\hline $22 \mathrm{~B}$ & 3 & ${ }^{3} \mathrm{~A}$ & 153.97 & 16.75 & 151.43 & 16.02 \\
\hline $23 \mathrm{~B}$ & 1 & ${ }^{1} \mathrm{~A}$ & 134.81 & 0.00 & 136.63 & 0.00 \\
\hline $23 \mathrm{~B}$ & 3 & ${ }^{3} \mathrm{~A}$ & 173.49 & 38.67 & 176.92 & 40.30 \\
\hline $24 \mathrm{~B}$ & 1 & ${ }^{1} \mathrm{~A}$ & 142.77 & 0.00 & 136.87 & 0.00 \\
\hline $24 \mathrm{~B}$ & 3 & ${ }^{3} \mathrm{~A}$ & 149.68 & 6.92 & 142.92 & 6.05 \\
\hline $25 \mathrm{~B}$ & 1 & ${ }^{1} \mathrm{~A}$ & 135.09 & 0.00 & 136.95 & 0.00 \\
\hline $25 \mathrm{~B}$ & 3 & ${ }^{3} \mathrm{~A}$ & 176.05 & 40.97 & 179.72 & 42.77 \\
\hline
\end{tabular}




\begin{tabular}{|c|c|c|c|c|c|c|}
\hline $26 \mathrm{~B}$ & 1 & ${ }^{1} \mathrm{~A}$ & 143.51 & 0.00 & 141.84 & 0.00 \\
\hline $26 \mathrm{~B}$ & 3 & ${ }^{3} \mathrm{~A}$ & 150.91 & 7.41 & 148.27 & 6.42 \\
\hline $27 \mathrm{~B}$ & 1 & ${ }^{1} \mathrm{~A}$ & 155.02 & 0.00 & 148.47 & 0.00 \\
\hline $27 \mathrm{~B}$ & 3 & ${ }^{3} \mathrm{~A}$ & 173.78 & 18.76 & 167.14 & 18.67 \\
\hline $28 \mathrm{~B}$ & 1 & ${ }^{1} \mathrm{~A}$ & 152.95 & 0.00 & 154.55 & 0.00 \\
\hline $28 \mathrm{~B}$ & 3 & ${ }^{3} \mathrm{~A}$ & 169.35 & 16.41 & 170.15 & 15.61 \\
\hline $29 \mathrm{~B}$ & 1 & ${ }^{1} \mathrm{~A}$ & 161.12 & 0.22 & 156.59 & 1.08 \\
\hline $29 \mathrm{~B}$ & 3 & ${ }^{3} \mathrm{~A}$ & 160.90 & 0.00 & 155.51 & 0.00 \\
\hline $29 \mathrm{~B}$ & 5 & ${ }^{5} \mathrm{~A}$ & 177.18 & 16.28 & 171.33 & 15.82 \\
\hline $30 \mathrm{~B}$ & 1 & ${ }^{1} \mathrm{~A}$ & 163.54 & 0.00 & 158.73 & 0.00 \\
\hline $30 \mathrm{~B}$ & 3 & ${ }^{3} \mathrm{~A}$ & 171.93 & 8.40 & 165.94 & 7.20 \\
\hline
\end{tabular}


Table S3. The relative energies $(\Delta \mathrm{E} 1, \mathrm{kcal} / \mathrm{mol})$ compared to the most stable isomers and the relative energies $(\Delta \mathrm{E} 2$, $\mathrm{kcal} / \mathrm{mol})$ between different multiplicities of each isomer for $\mathrm{UC}_{12}$ at B3PW91/(U: SDD; C: 6-31+G(d)) and PBE0/(U: SDD; C: 6-31+G(d)) two levels.

\begin{tabular}{|c|c|c|c|c|c|c|}
\hline \multirow{2}{*}{$\mathrm{UC}_{12}$} & \multirow{2}{*}{$2 \mathrm{~S}+1$} & \multirow{2}{*}{ State } & \multicolumn{2}{|c|}{ B3PW91 } & \multicolumn{2}{|c|}{ PBE0 } \\
\hline & & & $\Delta \mathrm{E} 1$ & $\Delta \mathrm{E} 2$ & $\Delta \mathrm{E} 1$ & $\Delta \mathrm{E} 2$ \\
\hline $1 \mathrm{C}$ & 3 & ${ }^{3} \mathrm{~A}$ & 0.00 & 0.00 & 0.00 & 0.00 \\
\hline $1 \mathrm{C}$ & 1 & ${ }^{1} \mathrm{~A}$ & 33.20 & 33.20 & 27.77 & 27.77 \\
\hline $1 \mathrm{C}$ & 5 & ${ }^{5} \mathrm{~A}$ & 29.06 & 29.06 & 28.76 & 28.76 \\
\hline $2 \mathrm{C}$ & 1 & ${ }^{1} \mathrm{~A}$ & 68.66 & 24.69 & 69.87 & 26.73 \\
\hline $2 \mathrm{C}$ & 3 & ${ }^{3} \mathrm{~A}$ & 43.97 & 0.00 & 43.14 & 0.00 \\
\hline $2 \mathrm{C}$ & 5 & ${ }^{5} \mathrm{~A}$ & 67.41 & 23.45 & 66.64 & 23.50 \\
\hline $3 \mathrm{C}$ & 1 & ${ }^{1} \mathrm{~A}$ & 97.56 & 27.07 & 103.33 & 27.17 \\
\hline $3 \mathrm{C}$ & 3 & ${ }^{3} \mathrm{~A}$ & 76.30 & 5.80 & 81.98 & 5.82 \\
\hline $3 \mathrm{C}$ & 5 & ${ }^{5} \mathrm{~A}$ & 70.49 & 0.00 & 76.16 & 0.00 \\
\hline $3 \mathrm{C}$ & 7 & ${ }^{7} \mathrm{~A}$ & 95.36 & 24.86 & 101.42 & 25.27 \\
\hline $4 \mathrm{C}$ & 1 & ${ }^{1} \mathrm{~A}$ & 93.11 & 17.27 & 98.06 & 19.09 \\
\hline $4 \mathrm{C}$ & 3 & ${ }^{3} \mathrm{~A}$ & 75.84 & 0.00 & 78.97 & 0.00 \\
\hline $4 \mathrm{C}$ & 5 & ${ }^{5} \mathrm{~A}$ & 112.08 & 36.24 & 115.94 & 36.98 \\
\hline $5 \mathrm{C}$ & 1 & ${ }^{1} \mathrm{~A}$ & 111.75 & 32.62 & 116.91 & 33.13 \\
\hline $5 \mathrm{C}$ & 3 & ${ }^{3} \mathrm{~A}$ & 79.13 & 0.00 & 83.78 & 0.00 \\
\hline $5 \mathrm{C}$ & 5 & ${ }^{5} \mathrm{~A}$ & 109.68 & 30.55 & 113.54 & 29.76 \\
\hline $6 \mathrm{C}$ & 1 & ${ }^{1} \mathrm{~A}$ & 116.17 & 27.34 & 120.66 & 34.70 \\
\hline $6 \mathrm{C}$ & 3 & ${ }^{3} \mathrm{~A}$ & 88.83 & 0.00 & 86.24 & 0.28 \\
\hline $6 \mathrm{C}$ & 5 & ${ }^{5} \mathrm{~A}$ & 93.95 & 5.12 & 85.97 & 0.00 \\
\hline $6 \mathrm{C}$ & 7 & ${ }^{7} \mathrm{~A}$ & 116.64 & 27.81 & 111.73 & 25.76 \\
\hline $7 \mathrm{C}$ & 1 & ${ }^{1} \mathrm{~A}$ & 110.73 & 1.87 & 111.20 & 7.79 \\
\hline $7 \mathrm{C}$ & 3 & ${ }^{3} \mathrm{~A}$ & 108.85 & 0.00 & 103.41 & 0.00 \\
\hline $7 \mathrm{C}$ & 5 & ${ }^{5} \mathrm{~A}$ & 115.59 & 7.14 & 110.27 & 3.93 \\
\hline $8 \mathrm{C}$ & 1 & ${ }^{1} \mathrm{~A}$ & 124.95 & 13.00 & 121.71 & 18.91 \\
\hline $8 \mathrm{C}$ & 3 & ${ }^{3} \mathrm{~A}^{\prime \prime}$ & 114.64 & 2.69 & 129.76 & 26.96 \\
\hline $8 \mathrm{C}$ & 5 & ${ }^{5} \mathrm{~A}$ & 111.95 & 0.00 & 102.80 & 0.00 \\
\hline $8 \mathrm{C}$ & 7 & ${ }^{7} \mathrm{~A}$ & 124.83 & 12.88 & 117.57 & 14.77 \\
\hline
\end{tabular}




\begin{tabular}{|c|c|c|c|c|c|c|}
\hline $9 \mathrm{C}$ & 1 & ${ }^{1} \mathrm{~A}$ & 117.13 & 9.05 & 116.00 & 12.24 \\
\hline $9 \mathrm{C}$ & 3 & ${ }^{3} \mathrm{~A}^{\prime \prime}$ & 109.69 & 1.61 & 108.78 & 5.02 \\
\hline $9 \mathrm{C}$ & 5 & ${ }^{5} \mathrm{~A}$ & 108.08 & 0.00 & 103.76 & 0.00 \\
\hline $9 \mathrm{C}$ & 7 & ${ }^{7} \mathrm{~A}$ & 121.71 & 13.62 & 116.67 & 12.90 \\
\hline $10 \mathrm{C}$ & 1 & ${ }^{1} \mathrm{~A}$ & 136.83 & 28.34 & 134.34 & 29.89 \\
\hline $10 \mathrm{C}$ & 3 & ${ }^{3} \mathrm{~A}$ & 108.49 & 0.00 & 104.45 & 0.00 \\
\hline $10 \mathrm{C}$ & 5 & ${ }^{5} \mathrm{~A}$ & 131.27 & 22.78 & 127.13 & 22.67 \\
\hline $11 \mathrm{C}$ & 1 & ${ }^{1} \mathrm{~A}$ & 121.56 & 14.41 & 122.72 & 9.26 \\
\hline $11 \mathrm{C}$ & 3 & ${ }^{3} \mathrm{~A}$ & 107.15 & 0.00 & 113.46 & 0.00 \\
\hline $11 \mathrm{C}$ & 5 & ${ }^{5} \mathrm{~A}$ & 114.01 & 6.87 & 116.50 & 3.04 \\
\hline $12 \mathrm{C}$ & 1 & ${ }^{1} \mathrm{~A}$ & 177.19 & 60.62 & 176.28 & 62.27 \\
\hline $12 \mathrm{C}$ & 3 & ${ }^{3} \mathrm{~A}_{2}$ & 151.69 & 35.13 & 147.19 & 33.19 \\
\hline $12 \mathrm{C}$ & 5 & ${ }^{5} \mathrm{~A}$ & 116.57 & 0.00 & 114.00 & 0.00 \\
\hline $12 \mathrm{C}$ & 7 & ${ }^{7} \mathrm{~A}$ & 146.05 & 29.48 & 143.46 & 29.45 \\
\hline $13 \mathrm{C}$ & 1 & ${ }^{1} \mathrm{~A}$ & 118.20 & 5.49 & 125.49 & 7.53 \\
\hline $13 \mathrm{C}$ & 3 & ${ }^{3} \mathrm{~A}$ & 112.70 & 0.00 & 117.96 & 0.00 \\
\hline $13 \mathrm{C}$ & 5 & ${ }^{5} \mathrm{~A}$ & 119.07 & 6.37 & 122.61 & 4.65 \\
\hline $14 \mathrm{C}$ & 1 & ${ }^{1} \mathrm{~A}$ & 154.67 & 24.62 & 151.40 & 27.49 \\
\hline $14 \mathrm{C}$ & 3 & ${ }^{3} \mathrm{~A}^{\prime}$ & 130.05 & 0.00 & 123.92 & 0.00 \\
\hline $14 \mathrm{C}$ & 5 & ${ }^{5} \mathrm{~A}$ & 141.93 & 11.87 & 134.17 & 10.25 \\
\hline $15 \mathrm{C}$ & 1 & ${ }^{1} \mathrm{~A}$ & 161.32 & 27.56 & 157.49 & 30.37 \\
\hline $15 \mathrm{C}$ & 3 & ${ }^{3} \mathrm{~A}$ & 133.76 & 0.00 & 127.13 & 0.00 \\
\hline $15 \mathrm{C}$ & 5 & ${ }^{5} \mathrm{~A}$ & 134.42 & 0.66 & 127.60 & 0.47 \\
\hline $16 \mathrm{C}$ & 1 & ${ }^{1} \mathrm{~A}$ & 164.25 & 30.67 & 162.12 & 32.96 \\
\hline $16 \mathrm{C}$ & 3 & ${ }^{3} \mathrm{~A}$ & 133.79 & 0.20 & 129.36 & 0.20 \\
\hline $16 \mathrm{C}$ & 5 & ${ }^{5} \mathrm{~A}$ & 133.58 & 0.00 & 129.16 & 0.00 \\
\hline $16 \mathrm{C}$ & 7 & ${ }^{7} \mathrm{~A}$ & 136.16 & 2.57 & 130.94 & 1.78 \\
\hline $17 \mathrm{C}$ & 1 & ${ }^{1} \mathrm{~A}$ & 148.91 & 11.03 & 146.78 & 14.67 \\
\hline $17 \mathrm{C}$ & 3 & ${ }^{3} \mathrm{~A}$ & 137.88 & 0.00 & 132.10 & 0.00 \\
\hline $17 \mathrm{C}$ & 5 & ${ }^{5} \mathrm{~A}$ & 146.08 & 8.20 & 139.91 & 7.81 \\
\hline $18 \mathrm{C}$ & 1 & ${ }^{1} \mathrm{~A}$ & 177.03 & 39.92 & 180.07 & 43.22 \\
\hline $18 \mathrm{C}$ & 3 & ${ }^{3} \mathrm{~B}_{1}$ & 140.75 & 3.63 & 156.83 & 19.97 \\
\hline
\end{tabular}




\begin{tabular}{|c|c|c|c|c|c|c|}
\hline $18 \mathrm{C}$ & 5 & ${ }^{5} \mathrm{~A}$ & 137.12 & 0.00 & 136.85 & 0.00 \\
\hline $18 \mathrm{C}$ & 7 & ${ }^{7} \mathrm{~A}$ & 138.49 & 1.37 & 138.54 & 1.69 \\
\hline $19 \mathrm{C}$ & 1 & ${ }^{1} \mathrm{~A}$ & 181.83 & 35.51 & 184.84 & 35.46 \\
\hline $19 \mathrm{C}$ & 3 & ${ }^{3} \mathrm{~A}$ & 146.31 & 0.00 & 149.38 & 0.00 \\
\hline $19 \mathrm{C}$ & 5 & ${ }^{5} \mathrm{~A}$ & 171.70 & 25.39 & 172.93 & 23.54 \\
\hline $20 \mathrm{C}$ & 1 & ${ }^{1} \mathrm{~A}$ & 218.11 & 59.66 & 218.46 & 62.68 \\
\hline $20 \mathrm{C}$ & 3 & ${ }^{3} \mathrm{~A}^{\prime}$ & 163.23 & 4.78 & 228.28 & 72.50 \\
\hline $20 \mathrm{C}$ & 5 & ${ }^{5} \mathrm{~A}$ & 158.45 & 0.00 & 155.78 & 0.00 \\
\hline $20 \mathrm{C}$ & 7 & ${ }^{7} \mathrm{~A}$ & 166.97 & 8.52 & 163.63 & 7.85 \\
\hline $21 \mathrm{C}$ & 1 & ${ }^{1} \mathrm{~A}$ & 179.88 & 16.20 & 177.79 & 19.95 \\
\hline $21 \mathrm{C}$ & 3 & ${ }^{3} \mathrm{~A}$ & 165.23 & 1.56 & 159.42 & 1.58 \\
\hline $21 \mathrm{C}$ & 5 & ${ }^{5} \mathrm{~A}$ & 163.67 & 0.00 & 157.84 & 0.00 \\
\hline $21 \mathrm{C}$ & 7 & ${ }^{7} \mathrm{~A}$ & 182.63 & 18.96 & 175.80 & 17.96 \\
\hline $22 \mathrm{C}$ & 1 & ${ }^{1} \mathrm{~A}$ & 164.75 & 0.00 & 163.17 & 3.88 \\
\hline $22 \mathrm{C}$ & 3 & ${ }^{3} \mathrm{~A}$ & 165.17 & 0.42 & 159.30 & 0.00 \\
\hline $22 \mathrm{C}$ & 5 & ${ }^{5} \mathrm{~A}$ & 165.50 & 0.75 & 160.79 & 1.49 \\
\hline $23 \mathrm{C}$ & 1 & ${ }^{1} \mathrm{~A}$ & 199.43 & 27.62 & 195.14 & 28.75 \\
\hline $23 \mathrm{C}$ & 3 & ${ }^{3} \mathrm{~A} "$ & 171.81 & 0.00 & 166.39 & 0.00 \\
\hline $23 \mathrm{C}$ & 5 & ${ }^{5} \mathrm{~A}$ & 182.52 & 10.71 & 180.97 & 14.58 \\
\hline $24 \mathrm{C}$ & 1 & ${ }^{1} \mathrm{~A}$ & 199.94 & 25.53 & 197.58 & 27.86 \\
\hline $24 \mathrm{C}$ & 3 & ${ }^{3} \mathrm{~A}$ & 174.41 & 0.00 & 169.72 & 0.00 \\
\hline $24 \mathrm{C}$ & 5 & ${ }^{5} \mathrm{~A}$ & 181.84 & 7.43 & 175.96 & 6.24 \\
\hline $25 \mathrm{C}$ & 1 & ${ }^{1} \mathrm{~A}$ & 199.94 & 25.53 & 198.81 & 28.33 \\
\hline $25 \mathrm{C}$ & 3 & ${ }^{3} \mathrm{~A}$ & 174.41 & 0.00 & 170.48 & 0.00 \\
\hline $25 \mathrm{C}$ & 5 & ${ }^{5} \mathrm{~A}$ & 181.84 & 7.43 & 175.98 & 5.50 \\
\hline $26 \mathrm{C}$ & 1 & ${ }^{1} \mathrm{~A}$ & 249.57 & 57.02 & 251.37 & 36.19 \\
\hline $26 \mathrm{C}$ & 3 & ${ }^{3} \mathrm{~A}$ & 192.55 & 0.00 & 215.19 & 0.00 \\
\hline $26 \mathrm{C}$ & 5 & ${ }^{5} \mathrm{~A}$ & 194.62 & 2.07 & 218.10 & -30.09 \\
\hline $27 \mathrm{C}$ & 1 & ${ }^{1} \mathrm{~A}$ & 185.97 & 81.71 & 187.02 & 0.00 \\
\hline $27 \mathrm{C}$ & 3 & ${ }^{3} \mathrm{~A}^{\prime}$ & 104.25 & 0.00 & 238.00 & 50.98 \\
\hline $27 \mathrm{C}$ & 5 & ${ }^{5} \mathrm{~A}$ & 104.48 & 0.23 & 246.07 & 59.05 \\
\hline $28 \mathrm{C}$ & 1 & ${ }^{1} \mathrm{~A}$ & 231.62 & 27.64 & 229.25 & 27.75 \\
\hline
\end{tabular}




\begin{tabular}{|l|l|l|l|l|l|l|}
\hline $28 \mathrm{C}$ & 3 & ${ }^{3} \mathrm{~A}^{\prime}$ & 203.98 & 0.00 & 201.74 & 0.24 \\
\hline $28 \mathrm{C}$ & 5 & ${ }^{5} \mathrm{~A}$ & 206.39 & 2.42 & 201.50 & 0.00 \\
\hline $28 \mathrm{C}$ & 7 & ${ }^{7} \mathrm{~A}$ & 152.60 & 51.37 & 216.04 & 14.54 \\
\hline $29 \mathrm{C}$ & 1 & ${ }^{1} \mathrm{~A}$ & 246.28 & 42.00 & 229.23 & 25.84 \\
\hline $29 \mathrm{C}$ & 3 & ${ }^{3} \mathrm{~A}$ & 204.65 & 0.37 & 214.96 & 11.57 \\
\hline $29 \mathrm{C}$ & 5 & ${ }^{3} \mathrm{~A}$ & 204.28 & 0.00 & 203.39 & 0.00 \\
\hline $29 \mathrm{C}$ & 7 & ${ }^{3} \mathrm{~A}$ & 215.56 & 11.28 & 219.98 & 16.59 \\
\hline $30 \mathrm{C}$ & 1 & ${ }^{1} \mathrm{~A}$ & 226.48 & 11.83 & 223.72 & 15.94 \\
\hline $30 \mathrm{C}$ & 3 & ${ }^{3} \mathrm{~A}$ & 214.65 & 0.00 & 207.79 & 0.00 \\
\hline $30 \mathrm{C}$ & 5 & ${ }^{5} \mathrm{~A}$ & 220.19 & 5.54 & 212.63 & 4.84 \\
\hline
\end{tabular}


Table S4. Relative energies ( $\Delta \mathrm{E}, \mathrm{kcal} / \mathrm{mol})$ for different spin states of $\mathbf{1 B}$ and $\mathbf{1 C}$.

\begin{tabular}{|c|c|c|c|c|c|}
\hline Species & \multicolumn{2}{|c|}{ 1B } & \multicolumn{3}{|c|}{ 1C } \\
\hline $2 S+1$ & 1 & 3 & 1 & 3 & 5 \\
\hline B3PW91/6-31+G(d) & 0.00 & 22.17 & 33.20 & 0.00 & 29.06 \\
\hline $\mathrm{PBE} 0 / 6-31+\mathrm{G}(\mathrm{d})$ & 0.00 & 22.34 & 27.77 & 0.00 & 28.76 \\
\hline B3PW91-D3/def2-TZVPP & 0.00 & 22.05 & 25.75 & 0.00 & 28.32 \\
\hline PBE0-D3/def2-TZVPP & 0.00 & 22.97 & 18.18 & 0.00 & 17.83 \\
\hline DLPNO-CCSD(T)/def2-TZVP & 0.00 & 10.14 & 20.30 & 0.00 & 16.13 \\
\hline T1 diagnostic & 0.011 & 0.017 & 0.014 & 0.032 & 0.036 \\
\hline
\end{tabular}


Table S5. EDA analyses for $\mathrm{An} / \mathrm{An}^{4+}+\left[\mathrm{C}_{12}\right] /\left[\mathrm{C}_{12}\right]^{4-} \rightarrow\left[\mathrm{AnC}_{12}\right](\mathrm{An}=\mathrm{Th}, \mathrm{U})$ at the PBE0/TZ2P level of theory.

\begin{tabular}{|c|c|c|c|c|}
\hline $\begin{array}{c}\text { Interacting } \\
\text { fragments }\end{array}$ & {$[\mathrm{Th}]\left({ }^{1} \mathrm{~A}\right)+\left[\mathrm{C}_{12}\right]\left({ }^{1} \mathrm{~A}\right)$} & {$[\mathrm{Th}]^{4+}\left({ }^{1} \mathrm{~A}\right)+\left[\mathrm{C}_{12}\right]^{4-}\left({ }^{1} \mathrm{~A}\right)$} & {$[\mathrm{U}]\left({ }^{5} \mathrm{~A}\right)+\left[\mathrm{C}_{12}\right]\left({ }^{1} \mathrm{~A}\right)$} & {$\left[\begin{array}{c}{[\mathrm{U}]^{4+}\left({ }^{3} \mathrm{~A}\right)+\left[\mathrm{C}_{12}\right]^{4-}} \\
\left({ }^{1} \mathrm{~A}\right)\end{array}\right.$} \\
\hline$\Delta \mathrm{E}_{\text {Pauli }}$ & 1153.24 & 364.54 & 902.69 & 347.08 \\
\hline$\Delta \mathrm{E}_{\text {Elstat }}$ & -614.58 & -1728.20 & -496.42 & -1734.90 \\
\hline$\Delta \mathrm{E}_{\text {Steric }}$ & 538.67 & -1363.66 & 406.27 & -1387.82 \\
\hline$\Delta \mathrm{E}_{\text {Orb }}$ & -748.73 & -716.62 & -668.23 & -798.88 \\
\hline$\Delta \mathrm{E}_{\text {int }}$ & -246.06 & -2080.29 & -261.97 & -2186.71 \\
\hline
\end{tabular}


Table S6. The Mayer, Gopinathan-Jug (G-J) and Nalewajski-Mrozek (N-M) bond orders for C-C and An-C bonds, and the calculated charges on the metal and carbon atoms of the $\mathbf{1 A}, \mathbf{1 B}$ and $\mathbf{1 C}$ at the PBE0/TZ2P level.

\begin{tabular}{|c|c|c|c|c|c|c|c|}
\hline \multirow{2}{*}{ Bond order } & \multicolumn{3}{|c|}{$\mathrm{C}_{12}$} & \multicolumn{2}{|c|}{$\mathrm{ThC}_{12}$} & \multicolumn{2}{|c|}{$\mathrm{UC}_{12}$} \\
\hline & C-C' & & $\mathrm{C}^{\prime}-\mathrm{C}^{\prime \prime}$ & Th-C & C-C & U-C & C-C \\
\hline Mayer & 2.25 & & 1.36 & 0.31 & 1.69 & 0.39 & 1.66 \\
\hline G-J & 2.15 & & 1.29 & 0.24 & 1.58 & 0.43 & 1.57 \\
\hline N-M & 2.25 & & 1.34 & 0.35 & 1.67 & 0.58 & 1.65 \\
\hline Charge & $\mathrm{Q}(\mathrm{C})$ & $\mathrm{Q}\left(\mathrm{C}^{\prime}\right)$ & $\mathrm{Q}\left(\mathrm{C}^{\prime \prime}\right)$ & $\mathrm{Q}(\mathrm{Th})$ & $\mathrm{Q}(\mathrm{C})$ & $\mathrm{Q}(\mathrm{U})$ & $\mathrm{Q}(\mathrm{C})$ \\
\hline Mulliken & -0.10 & 0.10 & -0.10 & 1.60 & -0.13 & 1.04 & -0.09 \\
\hline Hireshfeld & -0.06 & 0.06 & -0.06 & 1.09 & -0.09 & 0.82 & -0.07 \\
\hline VDD & -0.11 & 0.11 & -0.11 & 0.72 & -0.06 & 0.54 & -0.05 \\
\hline
\end{tabular}




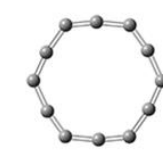

$1 \mathrm{~A} 0.00$

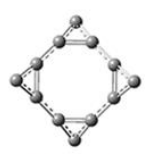

7 A 72.55

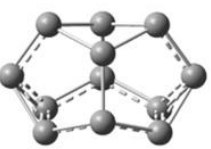

13A 106.86<smiles>CCCC1C(C)C1C</smiles>

19A 116.90

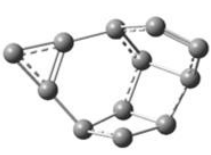

25A 135.37

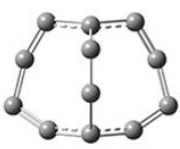

3A 57.80

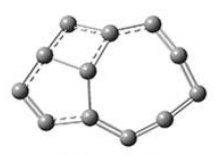

9A 84.54

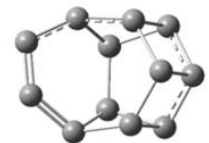

14A 107.22

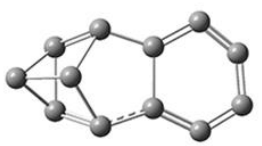

20A 116.90

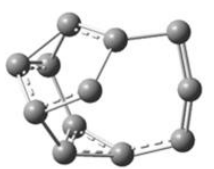

26A 139.19

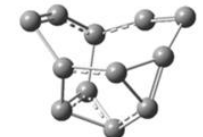

15A 108.77

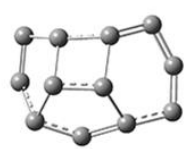

21A 121.62

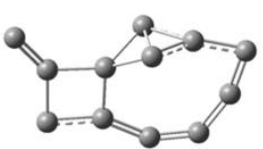

27A 139.39

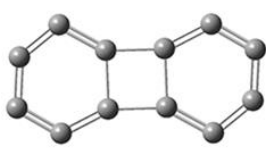

5A 60.97

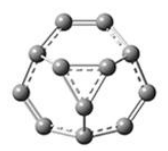

10A 85.31

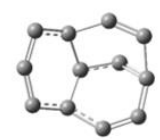

16A 112.84
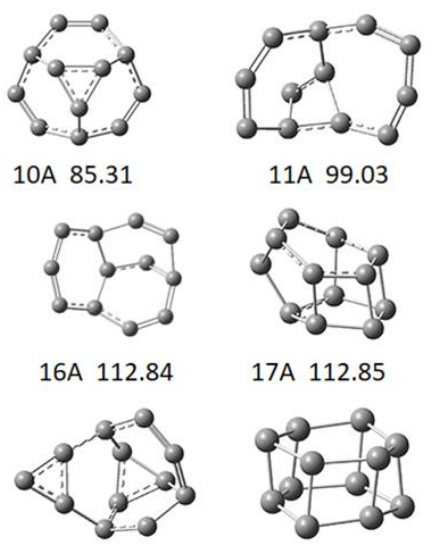

22A 121.93

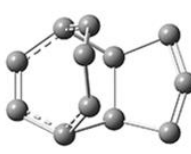

28A 142.51
11A 99.03

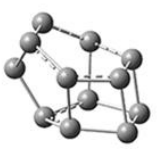

17A 112.85

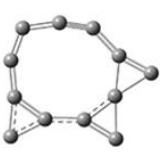

6A 67.59

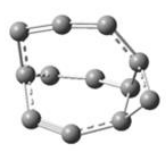

12A 106.51

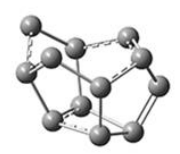

18A 113.78

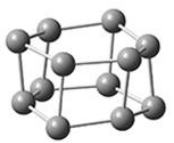

23A 128.46

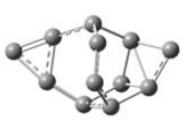

24A 133.60

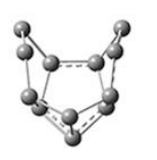

29A $146.76 \quad 30 A \quad 158.22$

Figure S1. The structures of most stable spin multiplicity and relative energies in $\mathrm{kcal} / \mathrm{mol}$ for the selected isomers of $\left[\mathrm{C}_{12}\right](\mathrm{A})$ at PBE0/6-31+G(d) level. 


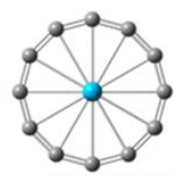

1B 0.00

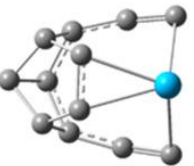

7B 89.43

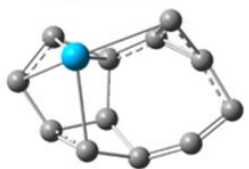

13B 119.28
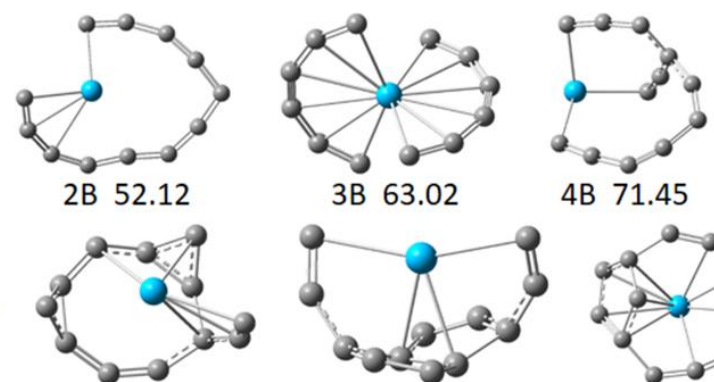

8B 97.73
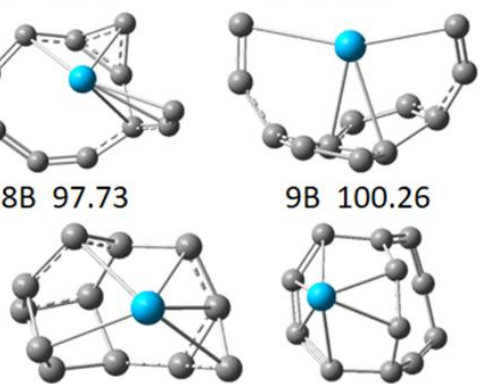

9B 100.26

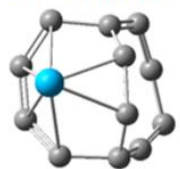

15B 122.66

14B 120.94

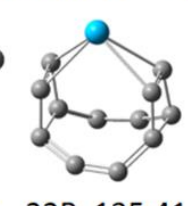

4B 71.45

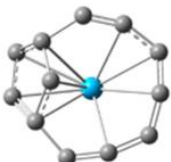

10B 102.97

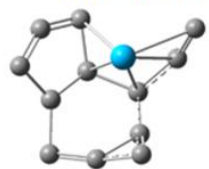

16B 124.33

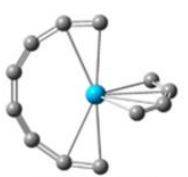

5B 75.31

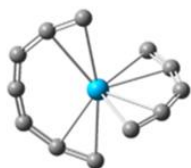

11B 104.85

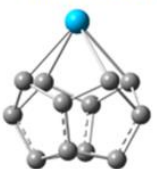

17B 125.11

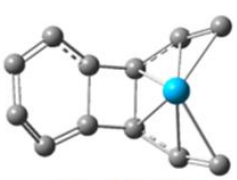

6B 80.44

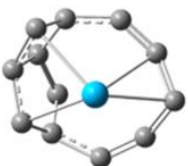

12B 106.91

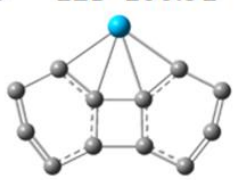

18B 125.67

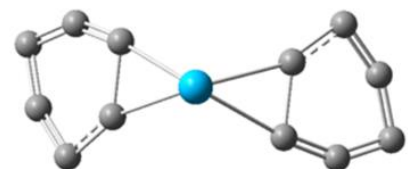

23B 136.63

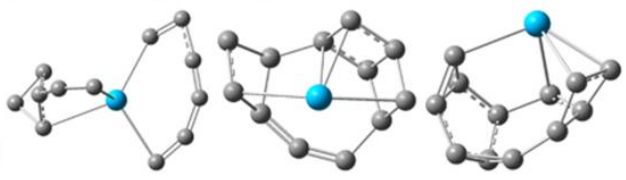

28B 154.55 29B $155.51 \quad 30 B 158.73$

Figure S2. The structures of most stable spin multiplicities and relative energies in $\mathrm{kcal} / \mathrm{mol}$ for the selected isomers of $\mathrm{ThC}_{12}(\mathbf{B})$ at PBE0/(Th: SDD; C: 6-31+G(d)) level. 


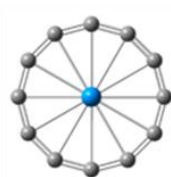

1C 0.00

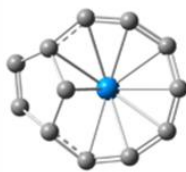

7C 99.47
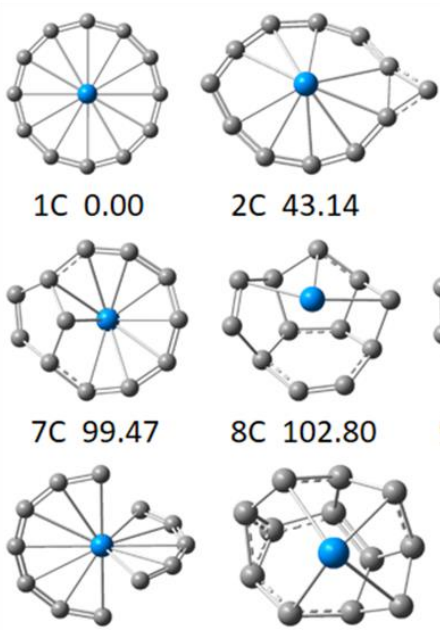

2C 43.14

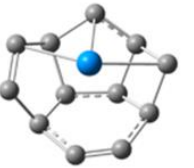

8C 102.80

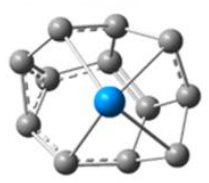

13C 117.96

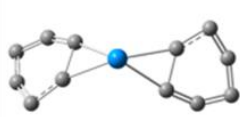

14C 123.92

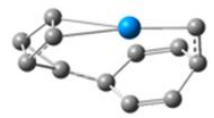

19C 149.38

20C 155.78

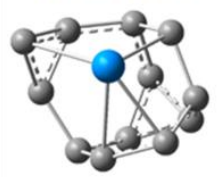

25C 170.48

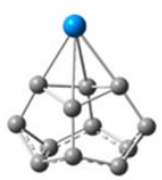

26C 185.10

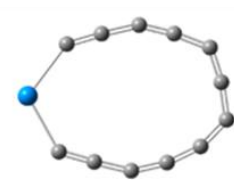

$3 C 76.16$

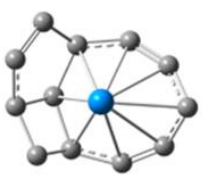

9C 103.76
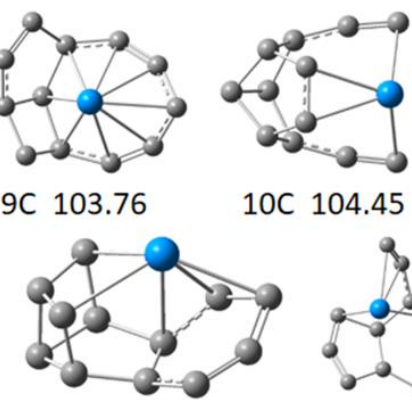

10C 104.45
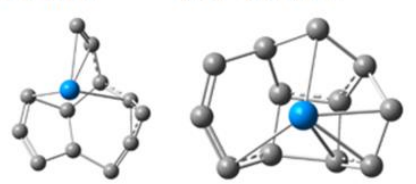

12C 114.00
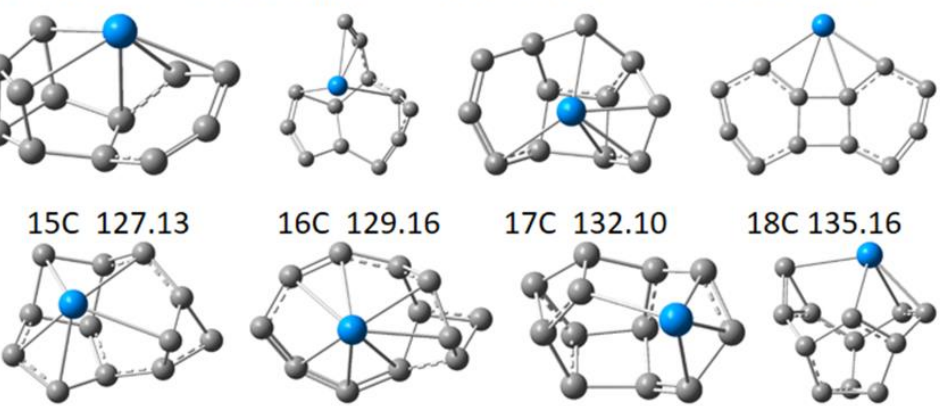

16C 129.16

17C 132.10

18C 135.16
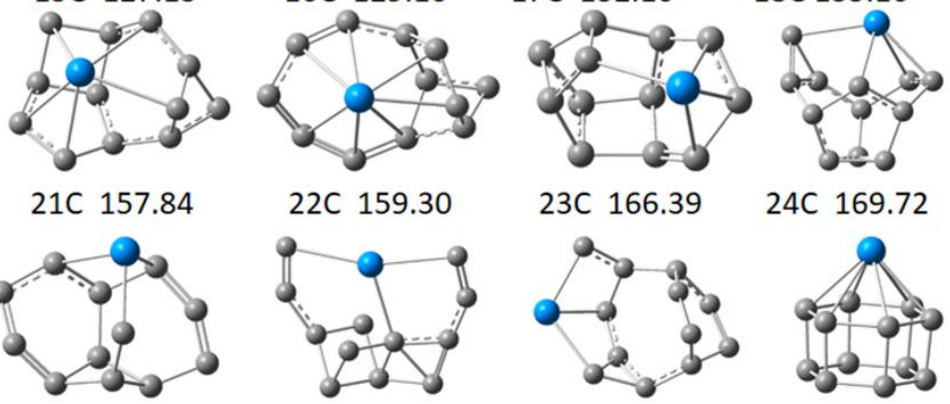

27C 187.02
22C 159.30

23C 166.39

24C 169.72

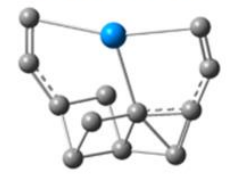

28C 201.50

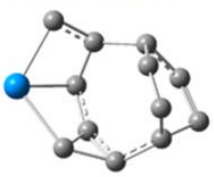

29C 203.39

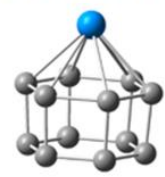

30C 207.79

Figure S3. The structures of most stable spin multiplicities, and relative energies in $\mathrm{kcal} / \mathrm{mol}$ for the selected isomers of $\mathrm{UC}_{12}(\mathbf{C})$ at PBE0/(U: SDD; C: 6-31+G(d)) level. 